\title{
Silver Nanoprisms and Nanotetrahedra Investigated by Transmission Electron Microscopy
}

\author{
Jian-Guo Zheng*
}

Electron Probe Instrumentation Center (EPIC)/NUANCE, Department of Materials Science \& Engineering, Northwestern University, Evanston, IL 60208

It has been found that the properties of nanomaterials are controlled by their size as well as by their shape [1-2]. Some methods were developed to make nanowires, nanobelts and nanodots [3-5]. Recently, a photoinduced method was invented to convert large quantities of silver nanospheres into triangular nanprisms which exhibit distinctive optical properties [2]. Using transmission electron microscopy (TEM), we have investigated the morphology and microstructures of the silver nanocrystals produced by the photoinduced method. These results may be useful to gain an insight into the growth mechanism and properties of the nanoparticles with the specific shape.

The specimens were prepared using the method described in reference 2. Figure 1 is a typical bright-field TEM micrograph of silver nanocrystals showing a triangular shape on carbon film. Most of these nanocrystals actually are triangular lamellae (called nanoprisms hereafter) with typical edge of $100 \mathrm{~nm}$ and thickness of $15 \mathrm{~nm}$. Electron diffraction patterns recorded from individual nanoprisms (Figs. 2a-b) indicate that they have an fcc crystal structure $(\mathrm{a}=0.408 \mathrm{~nm})$, and the normal of the triangular surface is parallel to the $<111>$ direction (Fig. 1a) and the edges the $<110>$ directions. Planar defects (twin boundary) exist in most of the nanoprisms and are parallel to the triangular surface. The additional weak spots in Fig. 2c, as compared with Fig. 2a, are attributed to the $\{111\}$ twin boundaries [6]. This is verified by tilting specimen to other zone axes, as shown in Fig. 2d, where two superimposed sets of diffraction spots resulting from the $<011>$ zone axis of the matrix and the $<411>$ zone axis of the twin are related by the $\{111\}$ twin boundary parallel to the triangular surface. Their corresponding spots are $02 \overline{2} \mathrm{~m} / 0 \overline{2} 2 t, \overline{3} 1 \overline{1} \mathrm{~m} / 1 \overline{3} \overline{1} t$ and $\overline{3} \overline{1} 1 \mathrm{~m} / 1 \overline{1} \overline{3} t$, where $m$ and $t$ represent matrix and twin, respectively.

Besides the nanoprisms, Ag nanotetrahedra have also been observed although they account for less than $1 \%$ of total particles. A bright field micrograph and its negative print (Fig. 3) show a triangular shape with clear thickness fringes, indicating the existence of Ag nanotetrahedra. Their surfaces are parallel to the $\{111\}$ lattice planes, which has been verified by tilting experiments.

In summary, Ag nanotetrahedra have been identified and Ag nanoprisms characterized using TEM. It may be interesting to produce large quantity of $\mathrm{Ag}$ nanotetrahedra.

[1] A.P. Alivisatos, Science, 271(1996), 933.

[2] R.C. Jin et. al., Science, 294(2001), 1901.

[3] J.T. Hu, T.W. Odom, C.M. Lieber, Acc. Chem. Res., 32(1999), 435.

[4] Z.W. Pan, Z.R. Dai, Z.L. Wang, Science, 291(2001), 1947.

[5] C.L. Haynes, R.P. Van Duyne, J..Phys. Chem. B 105(2001), 5599.

[6] A.I. Kirkland et.al., Proc. R. Soc. Lond. A 440(1993), 589-609.

* Correspondence: j-zheng3@northwestern.edu 


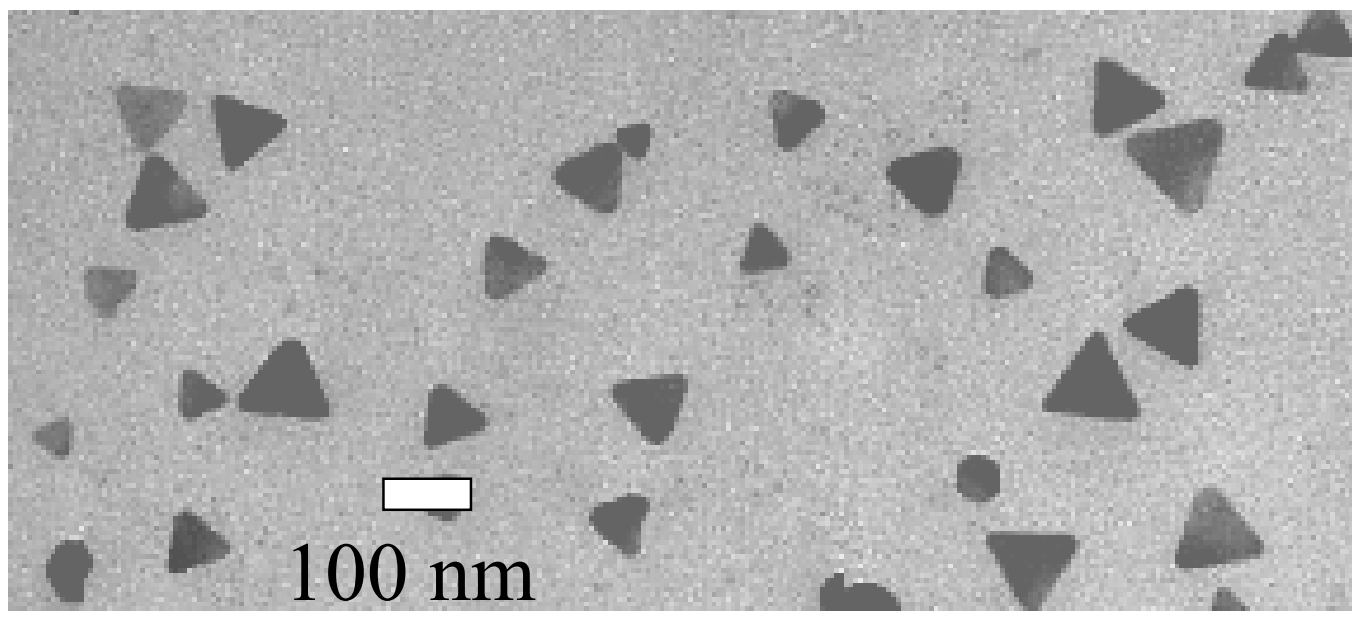

Figure 1. Bright-field TEM micrograph of Ag triangular particles on a carbon film

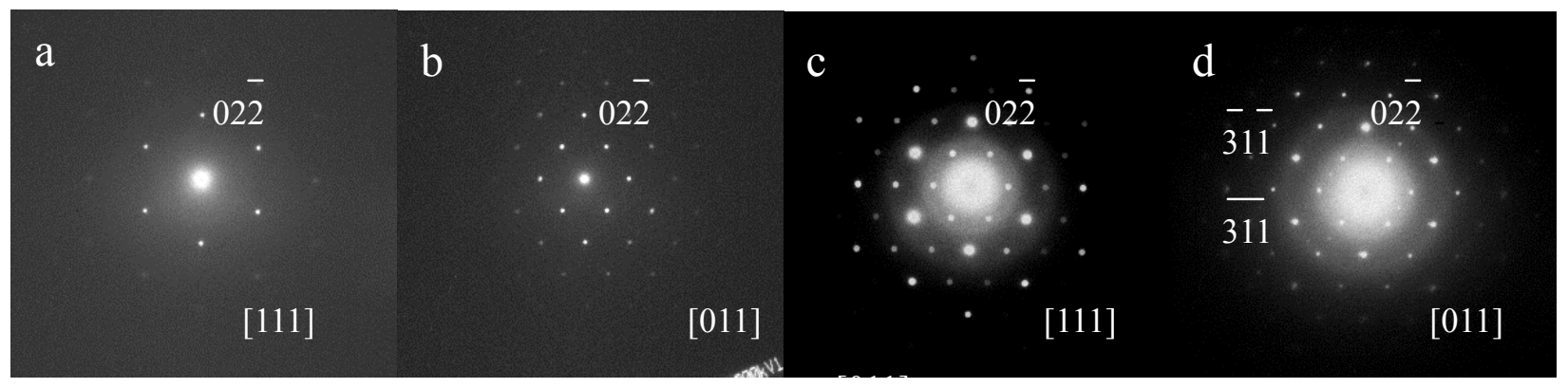

Figure 2. Typical diffraction patterns of Ag nanoprisms: (a)-(b) recorded from one nanoprism without defects, and (c)-(d) from one nanoprism with twin boundary.

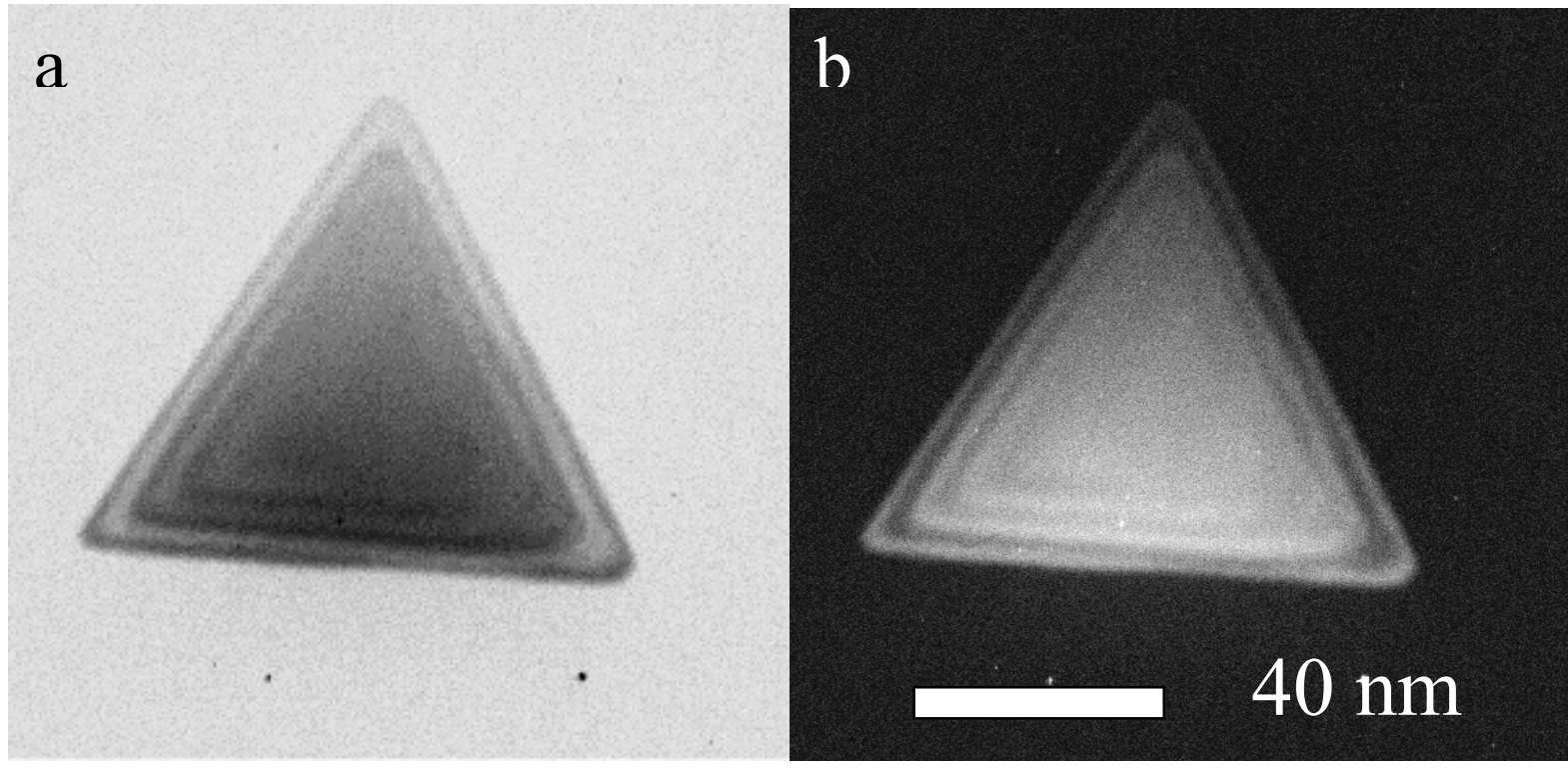

Figure 3. (a) Bright-field image of an Ag tetrahedron recorded when the electron beam is nearly parallel to the [111] axis. (b) the negative print of (a). 